\title{
Exchange-biased planar Hall effect sensor optimized for biosensor applications
}

\author{
Damsgaard, Christian Danvad; Freitas, S.C.; Freitas, P.P.; Hansen, Mikkel Fougt
}

Published in:

Journal of Applied Physics

Link to article, DOI:

$10.1063 / 1.2830008$

Publication date:

2008

Document Version

Publisher's PDF, also known as Version of record

Link back to DTU Orbit

Citation (APA):

Damsgaard, C. D., Freitas, S. C., Freitas, P. P., \& Hansen, M. F. (2008). Exchange-biased planar Hall effect sensor optimized for biosensor applications. Journal of Applied Physics, 103(7), 07 A302.

https://doi.org/10.1063/1.2830008

\section{General rights}

Copyright and moral rights for the publications made accessible in the public portal are retained by the authors and/or other copyright owners and it is a condition of accessing publications that users recognise and abide by the legal requirements associated with these rights.

- Users may download and print one copy of any publication from the public portal for the purpose of private study or research.

- You may not further distribute the material or use it for any profit-making activity or commercial gain

- You may freely distribute the URL identifying the publication in the public portal

If you believe that this document breaches copyright please contact us providing details, and we will remove access to the work immediately and investigate your claim. 


\title{
Exchange-biased planar Hall effect sensor optimized for biosensor applications
}

\author{
Christian D. Damsgaard, ${ }^{1, a)}$ Susana C. Freitas, ${ }^{2,3}$ Paulo P. Freitas, ${ }^{2,3}$ and \\ Mikkel F. Hansen ${ }^{1, b)}$ \\ ${ }^{1}$ MIC-Department of Micro and Nanotechnology, Technical University of Denmark, Building 345 East, \\ DK-2800 Kongens Lyngby, Denmark \\ ${ }^{2}$ INESC-Microsystems and Nanotechnologies, Rua Alves Redol, 9-1, 1000-029 Lisboa, Portugal \\ ${ }^{3}$ Physics Department, Instituto Superior Tecnico, Avenida Rovisco Pais, 1000 Lisbon, Portugal
}

(Presented on 6 November 2007; received 11 September 2007; accepted 5 October 2007; published online 23 January 2008)

This article presents experimental investigations of exchange-biased Permalloy planar Hall effect sensor crosses with a fixed active area of $w \times w=40 \times 40 \mu \mathrm{m}^{2}$ and Permalloy thicknesses of $t=20,30$, and $50 \mathrm{~nm}$. It is shown that a single domain model describes the system well and that the thicker film will have a higher signal as well as a lower noise. It is estimated that the signal-to-noise ratio for bead detection increases by a factor 2.1 when $t$ is increased from 20 to $50 \mathrm{~nm}$ and hence a higher $t$ is beneficial for biosensor applications. This is exemplified with calculations on M-280 Dynabeads®. (C) 2008 American Institute of Physics. [DOI: 10.1063/1.2830008]

\section{INTRODUCTION}

Magnetic biosensors rely on the combination of a sensitive magnetic field sensor with a bioassay that ensures specific binding of magnetic beads to the sensor surface when the target biological substance is present in the sample. ${ }^{1,2}$ Since the initial work of Schuhl et al., ${ }^{3}$ magnetic field sensors based on the planar Hall effect (PHE) have received considerable interest for low-noise magnetic biosensing., ${ }^{2,4-6}$ Exchange-biased PHE sensors with a $20 \mathrm{~nm}$ thick ferromagnetic (FM) layer were studied by Ejsing et al. ${ }^{4,5}$ Subsequently, the range of FM thicknesses from $t=3$ to $20 \mathrm{~nm}$ were studied by Tanh et al. ${ }^{6}$ We present investigations of exchange-biased Permalloy PHE sensor crosses with a fixed active area of $w \times w=40 \times 40 \mu \mathrm{m}^{2}$ and $t=20,30$, and $50 \mathrm{~nm}$. The investigations include the thickness dependence of sensor sensitivity, linear operation range, and electrical noise and it is shown that the sensor response to magnetic beads can be significantly improved by increasing $t$. The general results are exemplified by calculations of the signal-to-noise ratio (SNR) of a single M-280 Dynabead( ${ }^{\circledR}$ placed on top of the sensor center.

\section{SENSOR SIGNAL AND NOISE}

PHE sensors are based on the anisotropic magnetoresistance (AMR) observed in ferromagnetic materials. The AMR ratio is described by AMR $=\left(\rho_{\|}-\rho_{\perp}\right) / \rho$, where $\rho_{\|}$and $\rho_{\perp}$ are the resistivities parallel and perpendicular to the orientation of the magnetization, respectively, and $\rho=\left(\rho_{\|}+2 \rho_{\perp}\right) / 3$ is the average resistivity.

Figure 1 shows a micrograph of a sensor in which the geometric variables of the sensor are also defined. The sensor

\footnotetext{
a)Electronic mail: christian.damsgaard@mic.dtu.dk.

${ }^{b)}$ Electronic mail: mfh@mic.dtu.dk.
}

voltage $V_{y}$ depends on the current density $J_{x}$ along the $x$ axis and the angle $\theta$ between the magnetization $\mathbf{M}$ of the sensing area and $J_{x}$ as ${ }^{3}$

$$
V_{y}=J_{x} w\left(\rho_{\|}-\rho_{\perp}\right) \sin \theta \cos \theta .
$$

A well-defined initial state of $\mathbf{M}$ is established by exchange biasing the FM layer using an antiferromagnetic layer. The angle between the resulting easy direction and the $x$ axis, which is ideally zero, is called $\psi$. When a magnetic field $H_{y}$ is applied along the $y$ axis, the magnetization direction rotates by an angle $\theta$ with respect to the $x$ axis. This angle can be obtained by minimizing the energy density $u$, which for a single domain is

$$
\frac{u}{\mu M_{s}}=-H_{\mathrm{ex}} \cos (\theta-\psi)-\frac{1}{2} H_{K} \cos ^{2}(\theta-\psi)-H_{y} \sin \theta,
$$

where $M_{s}$ is the saturation magnetization, $H_{\mathrm{ex}}$ is the exchange field and $H_{K}$ is the anisotropy field. At low fields, the resulting sensor voltage is $V_{y}=S(t) H_{y}$ with the sensitivity

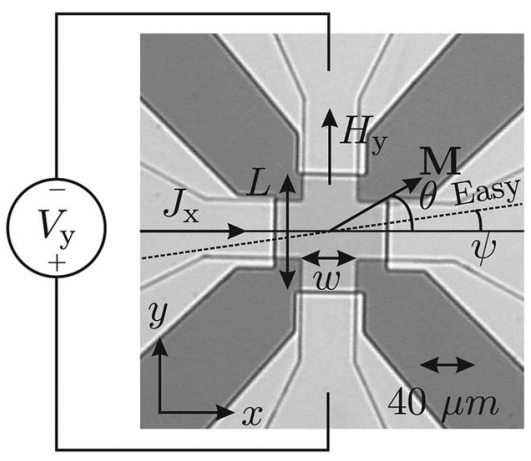

FIG. 1. Micrograph of the sensor cross with definitions of variables. The easy direction and easy axis are along the dashed line and form an angle $\psi$ to the $x$ axis. In the ideal case, $\psi=0$. 


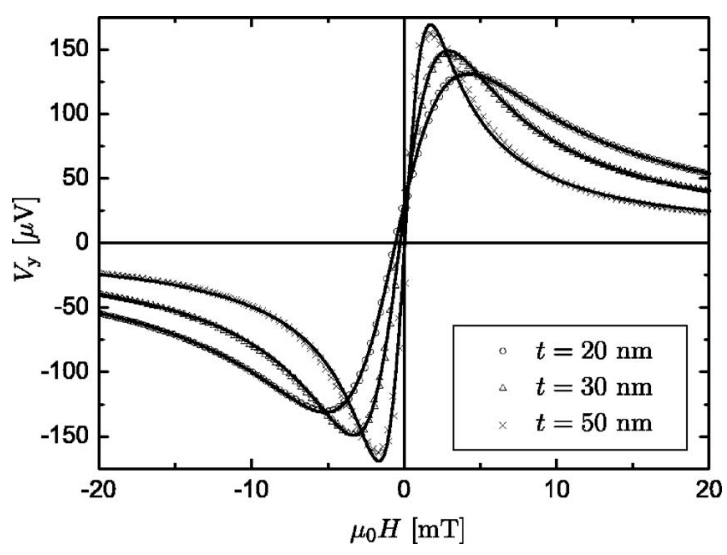

FIG. 2. PHE measurements (points) on sensors with $t=20,30$, and $50 \mathrm{~nm}$ as well as fits based on a single domain model (lines).

$$
S(t) \approx J_{x} w\left[\rho_{\|}(t)-\rho_{\perp}(t)\right] /\left[H_{\mathrm{ex}}(t)+H_{K}(t)\right] .
$$

For the investigated values of $t$, the thickness dependence is dominated by $H_{\mathrm{ex}}(t)$, which for a single domain decreases as $t^{-1}$. Moreover, the value of $\rho_{\|}(t)-\rho_{\perp}(t)$ increases slightly ${ }^{7}$ and $H_{K}(t)$ decreases for increasing $t$. Thus, all factors contribute to an increase of the sensor sensitivity for increasing $t$.

The model used for noise estimation consists of three contributions: $1 / f$ noise, thermal noise, and shot noise,

$$
\frac{\Delta V_{y}}{\sqrt{\Delta f}}=\frac{1}{\sqrt{t}}\left[\rho J_{x} \sqrt{\gamma_{H}\left(n_{c} f\right)^{-1}}+\sqrt{4 k_{B} T \rho \frac{L}{w}}+\rho \sqrt{2 e J_{x} w}\right],
$$

where $\Delta V_{y}$ is the noise, $\Delta f$ is the frequency bandwidth, $\gamma_{H}$ is Hooge's constant, $n_{c}$ is the number of charge carriers, $f$ is the frequency, $k_{B}$ is Boltzmann's constant, $T$ is the sample temperature, $L$ is the cross length, and $e$ is the elementary charge.

With parameters chosen realistically for our system $(\rho$ $=20 \mu \Omega \mathrm{cm}, \quad J_{x}=177 \mathrm{kA} / \mathrm{cm}^{2}, \quad \gamma_{H} \approx 10^{-2},{ }^{8} n_{c}=3.3$ $\times 10^{28} \mathrm{~m}^{-3},{ }^{9} T=300 \mathrm{~K}$ ), the noise is dominated by $1 / f$ noise at frequencies below $f \approx 10 \mathrm{~Hz}$, while above $f \approx 1 \mathrm{kHz}$ the noise characteristic is dominated by thermal noise. From Eq. (4), it is seen that the sensor noise is proportional to $t^{-1 / 2}$.

\section{EXPERIMENTAL DETAILS}

The top pinned magnetic stack $\mathrm{Ta}(3 \mathrm{~nm}) \backslash \mathrm{Ni}_{80} \mathrm{Fe}_{20}(t) \backslash$ $\mathrm{Mn}_{74} \operatorname{Ir}_{26}(20 \mathrm{~nm}) \backslash \mathrm{Ta}(3 \mathrm{~nm})$ was grown by ion-beam deposition on $\mathrm{Si}$ substrates with a $1 \mu \mathrm{m}$ thermal oxide using the same deposition conditions, as given in Ref. 7. During deposition, a magnetic field of $\mu_{0} H_{x}=4 \mathrm{mT}$ was applied ideally along the $x$ axis to define the easy axis and easy direction, but it could be misaligned with an angle $\psi$ (see Fig. 1). The sensor cross width $w=40 \mu \mathrm{m}$ and length $L=80 \mu \mathrm{m}$ were defined by the use of UV photolithography followed by ion milling. The $\mathrm{Ti}(10 \mathrm{~nm}) / \mathrm{Au}(20 \mathrm{~nm})$ contacts were subsequently defined by lift-off.

The electrical measurements were carried out using an ac current density $J_{x}=177 \mathrm{kA} / \mathrm{cm}^{2}(\mathrm{rms})$ applied along the $x$ axis at a frequency $f=2.2 \mathrm{kHz}$. The measurements were carried out pointwise in static external fields $\mu_{0} H_{y}$ swept be-
TABLE I. Values obtained from fits to electrical PHE measurements. The uncertainty estimates correspond to the $95 \%$ confidence intervals given by the least-squares fitting program.

\begin{tabular}{lcccc}
\hline \hline$t(\mathrm{~nm})$ & $\psi(\mathrm{deg})$ & $\mu_{0} H_{\mathrm{ex}}(\mathrm{mT})$ & $\mu_{0} H_{K}(\mathrm{mT})$ & $\rho_{\|}-\rho_{\perp}(\mu \Omega \mathrm{cm})$ \\
\hline 20 & $6.4(1)$ & $4.20(2)$ & $0.80(5)$ & $0.370(1)$ \\
30 & $4.5(2)$ & $2.64(2)$ & $0.66(4)$ & $0.420(1)$ \\
50 & $-1.6(4)$ & $1.43(2)$ & $0.38(5)$ & $0.480(3)$ \\
\hline \hline
\end{tabular}

tween $\pm 40 \mathrm{mT}$. The sensor voltage $V_{y}$ was measured by the use of lock-in technique. The presented data have been corrected for a voltage offset. All measurements were made at room temperature $T \approx 300 \mathrm{~K}$. The values of $\rho_{\|}$and $\rho_{\perp}$ were obtained from measurements on $50 \times 700 \mu \mathrm{m}^{2}$ Hall bars.

Magnetic hysteresis loops were measured in a LakeShore 7407 vibrating sample magnetometer (VSM) on photolithographically defined $3 \times 3 \mathrm{~mm}^{2}$ thin film samples.

\section{RESULTS AND DISCUSSION}

Figure 2 shows PHE measurements along with fits based on Eq. (1) and the single domain model [Eq. (2)]. The parameters resulting from the fits of the PHE measurements are given in Table I. Table II shows the corresponding values of $\psi, H_{\mathrm{ex}}$, and $H_{K}$ obtained from VSM measurements of easy axis hysteresis loops as well as $\rho_{\|}$and $\rho_{\perp}$ obtained from electrical measurements on Hall bars.

From Fig. 2, it is observed that the single domain model describes the system well. Comparing the values in Tables I and II, it is seen that the values of $\psi$ correspond to realistic misalignments of $J_{x}$ relative to the easy direction and that they show the same trend using the two techniques. Such a misalignment is mainly observed in the PHE measurements as a horizontal shift at $V_{y}=0 \mu \mathrm{V}$. Furthermore, the values of $H_{\text {ex }}$ obtained from fits of the PHE measurements on the sensor crosses are in excellent agreement with those obtained from the VSM measurements on the continuous films. In both cases, we find that $H_{\mathrm{ex}} \propto t^{-1}$ as expected. The values of $H_{K}$ obtained from the fits of the PHE measurements are systematically larger than those obtained from the VSM measurements. This can be due to the different types of measurements and that the anisotropy of the PHE sample may be influenced by its size and cross geometry. Finally, the values of $\rho_{\|}-\rho_{\perp}$ obtained from the fits of the PHE measurements agree with those obtained from Hall bar measurements within 9\%. The corresponding AMR values are in agreement with the findings of Gehanno et al. ${ }^{7}$ and for $t=50 \mathrm{~nm}$ the value of $2.4 \%$ calculated using the data in Table II is almost saturated at the value obtained for thick films. ${ }^{7}$

TABLE II. Values of $\psi, H_{\mathrm{ex}}$, and $H_{K}$ obtained from VSM measurements of easy axis hysteresis loops. The values of $\rho_{\perp}$ and $\rho_{\|}$are obtained from electrical measurements on Hall bars. The uncertainty estimates are based on repeated measurements.

\begin{tabular}{cccccc}
\hline \hline$t(\mathrm{~nm})$ & $\psi(\mathrm{deg})$ & $\mu_{0} H_{\mathrm{ex}}(\mathrm{mT})$ & $\mu_{0} H_{K}(\mathrm{mT})$ & $\rho_{\|}(\mu \Omega \mathrm{cm})$ & $\rho_{\perp}(\mu \Omega \mathrm{cm})$ \\
\hline 20 & $9(2)$ & $4.2(1)$ & $0.1(1)$ & $22.11(5)$ & $21.71(5)$ \\
30 & $6(2)$ & $2.7(1)$ & $0.3(1)$ & $21.70(5)$ & $21.24(5)$ \\
50 & $3(2)$ & $1.4(1)$ & $0.2(1)$ & $21.45(5)$ & $20.95(5)$ \\
\hline \hline
\end{tabular}


TABLE III. Calculated parameters for a single M-280 Dynabead ${ }^{\circledR}$ placed directly on top of the sensor center in an applied magnetic field corresponding to the negative limit of linear operation. The noise was estimated using Eq. (4) with the typical parameters listed in the text for $f=2.2 \mathrm{kHz}$ and $\Delta f=1 \mathrm{~Hz}$

\begin{tabular}{ccccc}
\hline \hline$t(\mathrm{~nm})$ & $\left\langle\mu_{0} H_{b}\right\rangle(\mathrm{nT})$ & $V_{y, b}(\mathrm{nV})$ & $\frac{\Delta V_{y}}{\sqrt{\Delta f}}(\mathrm{nV} / \sqrt{\mathrm{Hz}})$ & $\mathrm{SNR}$ \\
\hline \multirow{2}{*}{20} & 44.6 & 2.3 & 0.82 & 2.9 \\
30 & 29.9 & 2.7 & 0.67 & 4.0 \\
50 & 16.5 & 3.1 & 0.52 & 6.0 \\
\hline \hline
\end{tabular}

By analyzing the PHE data or, equivalently, using the fitting results, we find the low-field sensitivities $S(20 \mathrm{~nm})$ $=52.4 \mu \mathrm{V} / \mathrm{mT}, \quad S(30 \mathrm{~nm})=90.1 \mu \mathrm{V} / \mathrm{mT}, \quad$ and $\quad S(50 \mathrm{~nm})$ $=188 \mu \mathrm{V} / \mathrm{mT}$. The corresponding linear operation ranges within $2 \%$ are $\pm 0.73, \pm 0.49$, and $\pm 0.27 \mathrm{mT}$, respectively. The peaks of the sensor voltage are found to be 131, 149, and $161 \mu \mathrm{V}$, respectively.

All observations correspond well to the theoretical expectations for a single domain Permalloy film. When $t$ is increased from 20 to $50 \mathrm{~nm}$, the increase of the sensitivity by a factor of 3.6 is caused by the factor of 2.8 decrease of $H_{\mathrm{ex}}(t)+H_{K}(t)$ and the factor of 1.3 increase of $\rho_{\|}(t)-\rho_{\perp}(t)$ (cf. Table I). The increase in the maximum sensor voltage is solely due to the increase of $\rho_{\|}(t)-\rho_{\perp}(t)$, whereas the decrease in the linear operation range is solely due to the change of $H_{\mathrm{ex}}(t)+H_{K}(t)$. Thus, when used at the limit of the linear range, the signal is a factor of 1.3 higher for $t$ $=50 \mathrm{~nm}$ than for $t=20 \mathrm{~nm}$. The corresponding calculated noise decreases by a factor of 1.6 according to Eq. (4), resulting in a total increase of the SNR in this limit by a factor of $1.3 \times 1.6=2.1$. It is noted that if $t$ increases too much, $H_{K}$ will become larger than $H_{\mathrm{ex}}$ and the initial state of $\mathbf{M}$ along the easy direction will be lost. Moreover, the assumption of a single domain may not hold any longer.

As an example, we calculate the sensor response to a single M-280 Dynabead ${ }^{\circledR}$ with a bead radius of $R=1.4 \mu \mathrm{m}$ and magnetic susceptibility $\chi=0.76$ (Ref. 10) placed directly on top of the sensor center in an externally applied magnetic field at the negative limit of linear operation. The bead will give rise to an average positive dipole field $\left\langle\mu_{0} H_{b}\right\rangle$ on the active sensor area. Estimates of the signal due to a certain bead coverage are more complex and require detailed considerations of the sensor response versus bead position and sensor and bead parameters. ${ }^{11}$ Table III shows calculated values of $\left\langle\mu_{0} H_{b}\right\rangle$ as well as estimates of sensor signal $V_{y, b}$ due to the bead, electrical noise $\Delta V_{y} / \sqrt{\Delta f}$, and SNR. The changes of the values are in agreement with the general arguments given above. For $t=50 \mathrm{~nm}$, the calculations indicate a response to a single M-280 Dynabead ${ }^{\circledR}$ of $V_{y, b}=3.1 \mathrm{nV}$ with a SNR of 6.0. Thus, a single bead should easily be detectable on the sensor area. The sensor has space for $\sim 200$ beads and this reduces the influence of statistical fluctuations of the bead numbers in estimates of the bead coverage of the sensor.

\section{ACKNOWLEDGMENTS}

Financial support from the EU 6th Framework Programme BIODIAGNOSTICS project (Contract No. 017002) is acknowledged by C.D.D. and M.F.H.

${ }^{1}$ M. Megens and M. Prins, J. Magn. Magn. Mater. 293, 702 (2005).

${ }^{2}$ P. P. Freitas, H. A. Ferreira, D. L. Graham, L. A. Clarke, M. D. Amaral, V. Martins, L. Fonseca, and J. S. Cabral, in Magnetoelectronics: Magnetoresistive Biochips, edited by M. Johnson (Elsevier, Amsterdam, 2004).

${ }^{3}$ A. Schuhl, F. N. V. Dau, and J. R. Childress, Appl. Phys. Lett. 66, 2751 (1995).

${ }^{4}$ L. Ejsing, M. F. Hansen, A. K. Menon, H. A. Ferreira, D. L. Graham, and P. P. Freitas, Appl. Phys. Lett. 84, 4729 (2004).

${ }^{5}$ L. Ejsing, M. F. Hansen, A. K. Menon, H. A. Ferreira, D. L. Graham, and P. P. Freitas, J. Magn. Magn. Mater. 293, 677 (2005).

${ }^{6}$ N. T. Tanh, L. T. Tu, N. D. Ha, C. O. Kim, C. G. Kim, K. H. Shin, and B. P. Rao, J. Appl. Phys. 101, 053702 (2007).

${ }^{7}$ V. Gehanno, P. P. Freitas, A. Veloso, J. Ferreira, B. Almeida, J. B. Sousa, A. Kling, J. C. Soares, and M. F. da Silva, IEEE Trans. Magn. 35, 4361 (1999).

${ }^{8}$ R. van de Veerdonk, P. Belien, K. Schep, J. Kools, M. de Nooijer, M. Gijs, R. Coehoorn, and W. de Jonge, J. Appl. Phys. 82, 6152 (1997).

${ }^{9}$ S. Foner, Phys. Rev. 99, 1079 (1955).

${ }^{10}$ G. Fonnum, C. Johansson, A. Molteberg, S. Mørup, and E. Aksnes, J. Magn. Magn. Mater. 293, 41 (2005).

${ }^{11}$ C. D. Damsgaard and M. F. Hansen (unpublished). 